\title{
A functional-measurement study of apparent rarefaction
}

\author{
PAOLA BRESSAN, SERGIO C. MASIN, GIOVANNI VICARIO, and GIULIO VIDOTTO \\ University of Padua, Padua, Italy
}

\begin{abstract}
The apparent density of a group of elements (lines, dots, etc.) is affected by the area of the group itself: the smaller the area, the lower the density of the elements (apparent rarefaction). An explanation of apparent rarefaction, in terms of an averaging model, has been offered by Spinelli and Vicario (submitted for publication). If working in isolation, portions of the retina far from the center would contribute an apparent density different in magnitude from that contributed by the center. Thus, the overall apparent density would be a weighted average of the contributions due to the different portions of the retina. The averaging model has been tested here by functional-measurement methods. The results confirm the model.
\end{abstract}

"Apparent rarefaction" refers to the dependence of judgment of apparent visual density of elements in a group upon the area of the group itself. If two squares different in size are made up of lines, for example, and have the same physical density (lines per unit area), a lower apparent density is reported for the smaller square (Vicario, 1971a, 1971b, 1972). The effect is illustrated in Figure 1, where the small square appears to have sparser lines than the large square.

Spinelli and Vicario (submitted for publication) have proposed the following explanation of apparent rarefaction. Consider Figure 2a, representing a square made of lines tilted $45^{\circ}$. In Figure $2 \mathrm{~b}$, the same square is depicted in which a square contour delimiting a small area is added. Within the small area, the apparent density is less than the apparent density in the entire square of Figure 2a. According to Spinelli and Vicario, the visual system integrates information coming from a single retinal area delimited by a given proximal contour. The smaller area in Figure $2 b$ shows apparent rarefaction (if compared with the corresponding area in Figure 2a) only when the square contour is added; this fact suggests that the apparent density in Figure 2a is the result of an averaging of retinal information coming from two distinct areas (an area corresponding to the center of the retina, approximately framed by the small square contour in Figure $2 b$, and the remaining part of the square). More precisely, the retinal parts corresponding to central and peripheral vision would contribute two different apparent visual densities, $\mathrm{c}$ and $\mathrm{p}$, if stimulated separately. Because, in looking at Figure $2 \mathrm{a}$, both central and peripheral parts are stimulated without interruption (without any breaking contour), and a single visual density, $d$, is perceived, then $d$ must be a weighted average of $c$ and $p$. In symbols:

Requests for reprints may be addressed to any author at Dipartimento di Psicologia Generale, Piazza Capitaniato 3, 35139 Padova, Italy.

$$
\mathrm{d}=\mathrm{wc}+(1-\mathrm{w}) \mathrm{p},
$$

where $\mathrm{w}$ is a weight coefficient.

The structure of Model 1 may be checked through functional measurement (Anderson, 1981, 1982). The term c may be assumed to correspond to the apparent visual density produced by a very small area around the point of fixation. If the physical density of lines, and the side length of squares, like the one in Figure 2a, are varied factorially, for all the ensuing displays the same local informa-

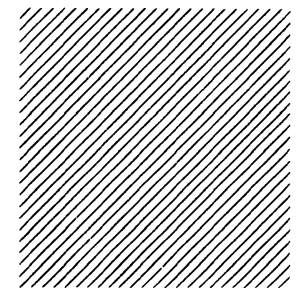

1a

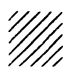

$1 \mathbf{b}$
Figure 1. Example of apparent rarefaction. The small square appears to have sparser lines than the large square (see also Vicario, 1971a).

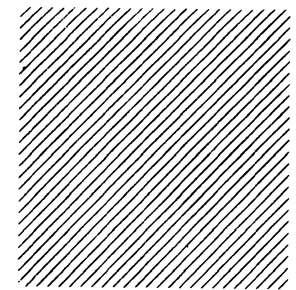

2a

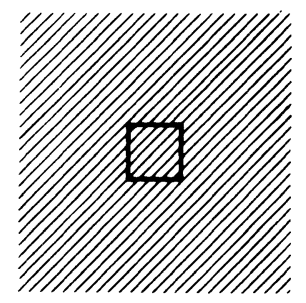

$\mathbf{2 b}$
Figure 2. Within the small area delimited by a square contour, the apparent density is less than that in the corresponding area of the square on the left. The reduction in density within an outlined area would suggest that different portions of the retina contribute differentially to the overall apparent density (Spinelli \& Vicario, submitted for publication). 
tion, corresponding to $\mathrm{c}$, is always sent to the central visual system. An increase in side length would contribute a progressive increase of $p$, and perhaps an increase in the weight that $p$ has. Given that the central information (c) is a constant, the overall density (d) is exclusively a function of the peripheral information (p) for a given weight (w). If side length is increased, then a graph, in which the parameter is physical density, should be a set of parallel curves expressing the relation of $d$ to side length.

\section{METHOD}

\section{Subjects}

Twenty-one university students with declared normal vision took part in the experiment.

\section{Stimuli}

The stimuli were the same as those used by Masin (1980). Square gratings made of parallel $45^{\circ}$-oblique black .5-mm lines (supplied by Mecanorma, Holland; sheet number 3125) were stuck in the center of $16 \times 16-\mathrm{cm}$ white sheets of cardboard (reflectance of about .9). Figure 1 illustrates two of these gratings. These squares represented a $5 \times 5$ (side length of a square $\times$ interlinear distance) factorial design, where for a side length of $2,4,6,8$, or $10 \mathrm{~cm}$ (subtending a visual angle of $2.6^{\circ}, 5.1^{\circ}, 7.6^{\circ}, 10.2^{\circ}$, or $12.7^{\circ}$, respectively, at a viewing distance of $45 \mathrm{~cm}$ ), the interlinear distance was $1.7,3.4,5.1,6.8$, or $10.2 \mathrm{~mm}$. The stimuli were illuminated by a $100-\mathrm{W}$ lamp. The illumination level was of $210 \mathrm{~lx}$.

\section{Procedure}

The subject sat at a small table provided with a head- and chinrest to keep the eyes level with the square presented on the frontoparallel plane. A random sequence of 25 squares was shown twice to each subject. Subjects were told to match numbers ranging between 0 and 100 to the density of lines in each square. Zero was defined as the smallest possible density corresponding to the limiting case where no line was in the square, and 100 was defined as the maximum density corresponding to the limiting case where the square was completely filled up by lines. The number 50 had to be assigned to the medium density. Other numbers had to be assigned in proportion to the density. The subject was asked to call out the number within $5 \mathrm{sec}$, and was told that, if he/she repented of the number just uttered, another number could be called out.

Before starting the experiment proper, five $16 \times 16$-cm squares with black $1-\mathrm{mm} 45^{\circ}$ tilted lines drawn over the entire square were shown for familiarization. The smallest density corresponded to 2 lines, and the highest density to 43 lines. Subjects learned how to assign the number by evaluating the density of lines in these squares once. After this preliminary task, the experiment proper began. A session lasted about $10 \mathrm{~min}$.

\section{RESULTS AND DISCUSSION}

The results are depicted in Figure 3. The mean rating of apparent density is represented on the ordinate. The side length is represented on the abscissa. The parameter is interlinear distance. The arithmetic mean of the two numbers called out by an observer for each grating was computed. Each filled circle in Figure 3 represents the arithmetic mean of the 21 means of these two numbers. A $5 \times 5$ ANOVA in which the factors were interlinear distance and side length was carried out. The main ef-

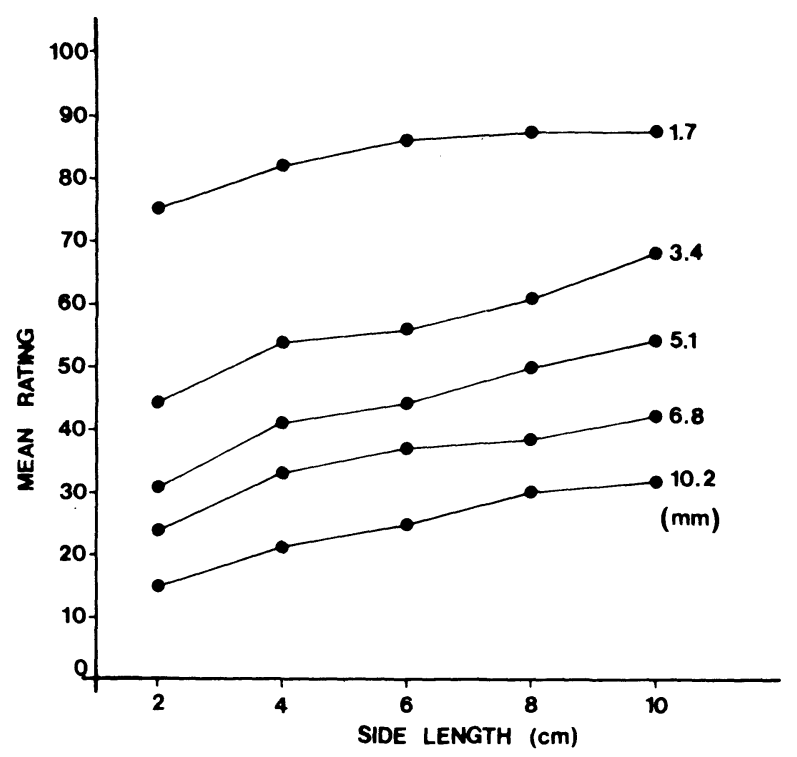

Figure 3. Graphic representation of the results of the present experiment. The side lengths $(\mathrm{cm})$ of squares are reported on the abscissa, and the mean ratings of apparent density are reported on the ordinate. The parameter is the distance $(\mathrm{mm})$ between lines in a square. The parallelism of curves, when the interlinear distance of $1.7 \mathrm{~mm}$ is excluded from the analysis, confirms Spinelli and Vicario's averaging model.

fects due to factor "side length" are statistically significant $[F(4,80)=41.6 ; p<.00005]$, thus confirming the existence of apparent rarefaction.

The interaction of factors is also statistically significant $[\mathrm{F}(16,320)=1.9, \mathrm{p}<.05]$. However, if a $4 \times 5$ analysis is carried out excluding the data for the interlinear distance $1.7 \mathrm{~mm}$ (upper curve in Figure 3), the interaction is not significant $[\mathrm{F}(12,240)=1.3]$.

The lack of interaction confirms Model 1-when the upper curve is excluded-in terms of information integration theory. This model does not apparently work for very small interlinear distances, as indicated by the "anomalous" behavior of the upper curve in Figure 3, and as confirmed by a previous finding (Masin, 1980). In other words, the upper curve seems to approach an asymptote for the gratings with a larger area. In these gratings the interlinear distances nearly equal the width of lines. So what makes these gratings somehow different from the others?

At present, we do not have any convincing answer. Yet, it might be observed that gratings with larger interlinear distances appear as black lines on a white background. As interlinear distances are made smaller and smaller, however, we get nearer to the limiting case in which the grating is perceived as a textured surface made up of alternating black and white lines. Reasonably, at this point the rating of the density of gratings may be based not so much on apparent density as on some other attribute of the whole textured surface, such as its compactness or its overall lightness. 


\section{REFERENCES}

ANDERSON, N. H. (1981). Foundations of information integration theory. New York: Academic Press.

ANDERSON, N. H. (1982). Methods of information integration theory. New York: Academic Press.

MASIN, S. C. (1980). A psychophysical study of apparent rarefaction. Perception, 9, 533-536.
SPINelli, D., \& Vicario, G. Apparent rarefaction: A tentative explanation. Submitted for publication.

VICARIO, G. (1971a). Un fenomeno di diradamento apparente in campo visivo. Archivio di Psicologia, Neurologia e Psichiatria, 32, 515-542.

VicArio, G. (1971b). Visual acuity and stimulus area. Psychologische Forschung, 35, 17-26.

VICARIO, G. (1972). Phenomenal rarefaction and visual acuity under 'illusory' conditions. Perception, 1, 475-482.

(Manuscript received for publication June 7, 1985.) 